Lo visible, lo invisible y las estrategias

Apropiación de tecnologías digitales por parte de jóvenes en organizaciones de la sociedad civil

María Florencia Pannunzio

Revista Argentina de Estudios de Juventud, (15), e062, 2021

ISSN 1852-4907 | https://doi.org/10.24215/18524907e062

https://perio.unlp.edu.ar/ojs/index.php/revistadejuventud

FPyCS | Universidad Nacional de La Plata

La Plata | Buenos Aires | Argentina

\title{
LO VISIBLE, LO INVISIBLE Y LAS ESTRATEGIAS
}

\section{APROPIACIÓN DE TECNOLOGÍAS DIGITALES POR PARTE DE JÓVENES EN ORGANIZACIONES DE LA SOCIEDAD CIVIL}

\author{
The Visible, the Invisible and the Strategies \\ Appropriation of Digital Technologies by Young People \\ in Civil Society Organizations
}

\section{María Florencia Pannunzio}

pannunzioflorencia@gmail.com

https://orcid.org/0000-0001-5587-1936

Centro de Estudios Sociales

Facultad de Humanidades

Universidad Nacional del Nordeste | Argentina

Palabras clave

jóvenes

Techo

OAJNU

tecnologías

Keywords

youth

Techo

OAJNU

technologies

\section{Resumen}

A partir de un abordaje cualitativo analizaremos las estrategias y las apropiaciones que jóvenes agrupados/as en las organizaciones civiles OAJNU y Techo realizaron de Facebook, Twitter e Instagram durante los años 2016-2017. Se identifica que en los usos constantes adquieren un conocimiento especializado que se vincula a la gestión, la pre-figuración de públicos específicos y las lógicas de publicación que cada una de las redes sociales digitales promueve, entre otros aprendizajes.

\section{Abstract}

From a qualitative approach, we will analyze the strategies and appropriations that young people members of civil organizations OAJNU and Techo carry out on Facebook, Twitter and Instagram during the period 2016-2017. We identified that in constant uses they acquire specialized knowledge about management, the pre-figuration of specific audiences and the logic of publication in each of the digital social networks, among other learning. 


\title{
LO VISIBLE, LO INVISIBLE Y LAS ESTRATEGIAS \\ APROPIACIÓN DE TECNOLOGÍAS DIGITALES \\ POR PARTE DE JÓVENES EN ORGANIZACIONES \\ DE LA SOCIEDAD CIVIL
}

\author{
Por María Florencia Pannunzio
}

\section{Introducción}

En este trabajo se analiza la apropiación de tecnologías digitales por parte de jóvenes en el marco de dos organizaciones de la sociedad civil (OSC) internacionales que tienen sede en la ciudad de Corrientes, Argentina: la Organización Argentina de Jóvenes para las Naciones Unidas (OAJNU) y Techo, las cuales agrupan mayoritariamente a jóvenes, a la vez que los condicionan y les imprimen un «saber hacer» particular. Hacemos referencia a que son organizaciones que condicionan, en tanto que ofrecen ciertos lineamientos de acción que deben ser seguidos y que actúan como orientadores en sus procesos de socialización política (Pannunzio, 2019).

Nos preguntamos, ¿qué aprendizajes obtienen en relación con las prácticas en los espacios digitales? El análisis se sustenta sobre la base de entrevistas semiestructuradas y de una sistematización de usos de redes sociales (Facebook, Twitter, Instagram) durante los años 2016 y 2017, realizadas en el marco de la tesis doctoral de la autora. ${ }^{2}$ Nos interesa indagar cuáles fueron los usos y las apropiaciones con vistas a convertir a la web en un espacio de acción y de organización social.

Nos posicionamos desde una perspectiva crítica, que atiende a una doble mediación, tecnológica y social, para identificar las estrategias, los posicionamientos y las formas de estar juntos/as-conectados/as. Como punto de partida, establecemos coordenadas teórico-metodológicas. A continuación, analizamos, por un lado, lo que aparece «visible» en sus redes, es decir, con carácter público y, por otro, lo que permanece «invisible», con carácter privado. Por último, nos detenemos en las estrategias y en la frecuencia de publicación para dar cuerpo a las apropiaciones que realizan de las Tecnologías de la Información y la Comunicación (TIC). 


\section{Coordenadas teórico-metodológicas}

Los/as jóvenes tienen en la web social su espacio de comunicación, de socialización y de acción privilegiado (Feixa \& Fernández Planells, 2014). Las tecnologías digitales les permiten contactarse con personas, instituciones, organismos y gobiernos más allá de las distancias o las cercanías geográficas. Se trata de un escenario con fronteras difusas, en tanto los momentos de conexión se superponen con las actividades de la vida cotidiana.

Cuando hablamos de apropiación, nos referimos al «conjunto de procesos socioculturales que intervienen en el uso, la socialización y la significación de las nuevas tecnologías en diversos grupos socioculturales» (Wincour, 2009, p. 20). Entendemos que la apropiación es un proceso simbólico y material por el cual un sujeto o un grupo social realizan, en primera instancia, una elucidación crítica del entramado mediático y tecnológico que tienen a su alcance, y, posteriormente, toman el contenido significativo de un artefacto y lo hacen propio. Lo dotan de sentido y lo incorporan a su vida cotidiana (Morales \& Loyola, 2013; Lemus, 2017).

Silvia Lago Martínez, Anahí Méndez y Martín Gendler (2017) plantean que el concepto de apropiación de tecnologías suele remitir a la práctica de «hacer propio lo ajeno» y coloca el foco en cómo se accede, se utiliza y se dota de sentido a las prácticas individuales y colectivas en relación con tecnologías, en el marco de contextos culturales, socioeconómicos e históricos diversos y desiguales. En este sentido, Delia Crovi Druetta (2013) plantea que «su significado cotidiano: adueñarse de algo, tomar para sí un objeto o recurso, lo que le pertenece a un sujeto, está lejos de explicar un fenómeno tan complejo» (p. 11). Cuando indagamos en las apropiaciones, nos interesan las prácticas que se habilitan o se clausuran a partir de ellas, así como los sentidos que se construyen, que casi siempre son compartidos y se insertan en las comunidades y/o en los grupos a los que pertenecemos.

La perspectiva crítica desde la cual nos posicionamos integra la doble mediación (social y tecnológica) y comprende, por lo tanto, el acceso a los dispositivos tecnológicos como un problema de recursos y de infraestructuras, pero también de desarrollo de habilidades tecnológicas y de capital cultural de cada individuo o comunidad para el aprovechamiento de las posibilidades que dichas tecnologías presentan (Crovi Druetta, 2013; Moreno Gálvez, 2015). 
Si se atiende a los procesos de apropiación, es posible identificar las características que adquiere el «mundo digital»: capacidad para moverse en una nueva dimensión espacio-temporal; creación y despliegue de actividades mediante comunidades virtuales; posibilidad de crear, de compartir y de intercambiar contenidos. En este sentido, la utilización de herramientas y de medios tecnológicos trae aparejados diversos cambios en los usos del lenguaje, tanto escrito como oral y audiovisual (Martín-Barbero, 2002). En lo que refiere a la web social, los modos de comunicación están fuertemente marcados por aspectos icónicos (emoticones, fotografías, videos, gifs, flyers) y por elementos que permiten la conectividad y la discusión temática y personalizada: nos referimos, específicamente, al uso del arroba (@), en tanto forma directa de interpelación, y del hashtag (\#), como unidad temática a partir de la cual se puede marcar agenda o bien sumarse a tendencias de conversación social (trending topics).

El hashtag en Twitter opera como una especie de cluster o racimo de nodos o lugares de enunciación articulados por el o los términos de referencia, en el que los usuarios del hashtag colaboran (y se encuentran), discrepan (y disputan sentidos) o atacan (descalifican, contraargumentan o amenazan) [Reguillo, 2017, p. 105].

Nuestros materiales de trabajo provienen, por un lado, de catorce entrevistas semiestructuradas a jóvenes de entre 18 y 29 años que ocupan distintos puestos o roles al interior de las organizaciones; es decir, no solo de aquellos/as vinculados/as al manejo de redes, a quienes presentamos con nombres ficticios. Por otro lado, de una sistematización realizada sobre los perfiles activos de ambas organizaciones: en Techo, un grupo en Facebook llamado «Voluntarios Techo Chaco-Corrientes»; en OAJNU, sus perfiles en Facebook (@OAJNUCtes), Instagram (/oajnucorrientes) y Twitter (@oajnucorrientes).

La sistematización de la información se realizó mediante un cuadro en el que se consignó: fecha, contenido y soportes (videos, flyers, fotos, textos, etc.); vínculos temporales (ahora, antes, ayer, mañana, más adelante) entre la publicación y las actividades; redes sociales de publicación y comentarios; y otras observaciones (cantidad de «Me gusta», de retuits y de comentarios obtenidos por la publicación). 


\section{Lo visible}

En Techo, la mayor parte de la información que se difunde sobre la organización es centralizada por la Coordinación Regional de Comunicación, ubicada en la Ciudad Autónoma de Buenos Aires (CABA), y si bien desde la sede pueden aportar contenidos no disponen de vía libre para publicar en nombre de la organización. Al respecto, Federico (31 años, Techo) plantea que, en líneas generales, «se decide todo allá», pero que en la sede se hacen cargo de la confección de flyers y de videos, así como del envío de mails masivos (mailing). Esta estructura verticalista de la comunicación también afecta la toma de decisiones, hasta el punto de no poder generar hashtag (\#) locales.

El grupo en Facebook, "Voluntarios Techo Chaco-Corrientes», tiene más de 2.600 miembros (números de diciembre 2017). ${ }^{3}$ «Hablamos mucho de este grupo porque en lo digital, salvando el tema de los correos, que mandamos muy pocos, es la única herramienta real que tenemos»(Federico, 31 años, Techo). Desde este espacio, difunden sus actividades (capacitaciones, colectas, talleres, cine-debate, charlas, intervenciones, etc.), buscan "captar» voluntarios/as y organizan campañas que sostienen a lo largo de varios meses, y cuyo eje está puesto en la inclusión de los sectores vulnerables y en la necesidad de involucrarse con el entorno, en particular, con los asentamientos.

De acuerdo con las condiciones que menciona Moreno Gálvez (2015), identificamos que en el uso repetitivo de las herramientas, y en pos de generar una comunidad virtual, se establecen acuerdos básicos sobre estilos de publicación, días y horarios, colores y palabras a utilizar, todo lo cual se encuentra pautado en el manual de marca de cada organización. Al respecto, Amelia (22 años, Techo) plantea que llegaron a definir criterios mediante prueba y error. El principal acuerdo es no saturar las redes y publicar dos o tres veces al día en los horarios en que las personas más se conectan, a las doce del mediodía y antes de acostarse.

En Techo, el uso de hashtags se define a nivel regional o nacional, según las campañas de comunicación de la organización. De este modo, se fueron asentando diversas frases que aluden a temáticas como hábitat, asentamientos, colecta, Relevamiento de Asentamientos Informales (RAl), al tiempo que presentan a la organización como un espacio de trabajo ameno y que invitan a sumarse (\#QuéGrandeEsteEquipo, \#OportunidadLaboral). 
A partir del relevamiento, se observa que fueron escasas las oportunidades en las que las organizaciones invitaron de manera explícita a movilizarse mediante hashtags para sentar presencia en el espacio digital. Un ejemplo excepcional se produjo a fines de 2016, cuando se iban a presentar en distintos puntos del país los resultados del RAI. Desde Techo, invitaban a tuitear:

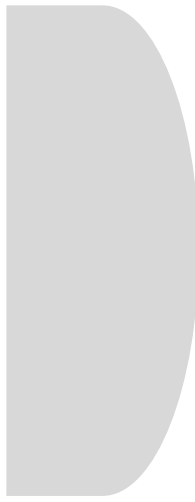

[...] Este lunes, a partir de las 2 de la tarde, queremos que durante la presentación del \#RelevamientoTECHO, todo el país nos escuche decir que la ciudad que tenemos, «no es una ciudad, si no es para todxs». Por eso te invitamos a twittear:

Corrientes no es una ciudad, si no es para todos \#RelevamientoTECHO

Resistencia no es una ciudad si no es para todos \#RelevamientoTECHO

O bien cualquier otra ciudad que quieras que sea para todos

(Techo, publicación en Facebook, 31/10/2016).

La sede Corrientes de OAJNU posee una fuerte presencia en tres redes sociales digitales: Facebook, Twitter e Instagram, las cuales fueron utilizadas de manera prácticamente diaria durante el período en que se realizó el relevamiento; en algunas jornadas, incluso, con más de una publicación. En febrero de 2016, habilitaron la función de vincular esta red con las cuentas de Facebook y de Twitter.

En mayo de ese mismo año se sumó Instagram, red social que incorpora las historias como un recurso narrativo en tiempo presente. Estas publicaciones permanecen durante 24 horas a la vista de los contactos y, a diferencia de las publicaciones en el perfil de usuario, las interacciones que suscitan (en términos de «me gusta» y de comentarios), permanecen en un plano privado. La incorporación de este nuevo perfil suscitó discusiones sobre qué tipos de usos otorgarle a nivel organizacional. Se acordó subir fotos de situaciones concretas, sin mucho texto, y con los hashtags que se emplean a nivel nacional.

Fue todo un debate cómo trabajar con las historias [de Instagram].

Si trabajar o no, y qué hacer, porque era todo muy nuevo. La mayoría las conocíamos, pero no a nivel organización [...]. A medida que aumentaron los seguidores, que no solo eran participantes sino gente común -incluso, funcionarios que siguen a la organización-, se comenzó a trabajar más con videos de los participantes hablando o de nosotros hablando... 
Por ejemplo, en Modelo Junior ${ }^{4}$ el Instagram se usó muchísimo, porque era como mostrar el detrás de escena de lo que estaba pasando y también era información para los participantes (Melina, 19 años, OAJNU, 2017).

Las imágenes y los textos que publican en esta red muestran a los/as jóvenes de OAJNU en un tono más intimista, y presentan a cada voluntario/a y a las redes que tejen desde la organización. También dan cuenta de las actividades de capacitación, entre las que se destacan: oratoria, negociación y coordinación de equipos. En este caso, se pueden ver las escuelas donde realizan todos sus proyectos, cómo son sus dinámicas de trabajo (en rondas, haciendo manualidades) y la masividad de los grupos con los que trabajan. Además, refuerzan el armado y la publicación de flyers que utilizan los colores de la organización.

Su página en Facebook es muy activa. Si bien hay un equipo de voluntarios/as constituido para hacerse cargo de la Secretaría de Comunicación, una práctica habitual durante las actividades masivas es que cualquier voluntario/a dispuesto/a a colaborar para mantener actualizadas las redes en tiempo presente (por ejemplo, contando en Twitter lo que sucede en una capacitación o compartiendo en Facebook y en Instagram fotos de distintos momentos de los Modelos de Naciones Unidas) tenga acceso a las contraseñas. De este modo, cuentan las actividades y las propuestas del día: talleres, reuniones, saludos por cumpleaños, bienvenida a nuevos/as voluntarios/as, invitaciones a sumarse, descripciones de los proyectos en curso, etcétera.

Ya adelantamos la función que cumplen en la construcción de presencia en entornos digitales los usos de herramientas como el Hashtag (\#) y el Arroba (@). Se trata de elementos que permiten nombrar, convocar, interpelar, agrupar, hacer llamados de atención, iniciar conversaciones, instalar temas en la agenda pública virtual. Rossana Reguillo (2017) los nombra como repertorios de la acción conectiva, entre los que incluye el uso de streaming, los memes, el micrófono humano ${ }^{5}$ y el uso de hashtags.

A diferencia de Techo, que no tiene permitido generar un hashtag propio de la sede CoCha (Corrientes/Chaco), en OAJNU no tienen esa restricción, aunque a nivel nacional se establecieron acuerdos cuyos lineamientos venían dados por el Plan Estratégico Organizacional 2014. Carolina (24 años, OAJNU) comenta que en ese documento se propusieron cuatro ejes, que se tradujeron en los cuatro hashtags 
que utilizan con más frecuencia: \#MásPares, \#MásVoces, \#MásFuerza \#MásDonantes. A lo largo de los encuentros, las reuniones y los plenarios realizados durante el año, siempre se realizan capacitaciones sobre el uso de estos recursos, en las que se remarca la necesidad de utilizar los hashtags como un modo de interpelar y de aumentar la identidad de la organización en las redes.

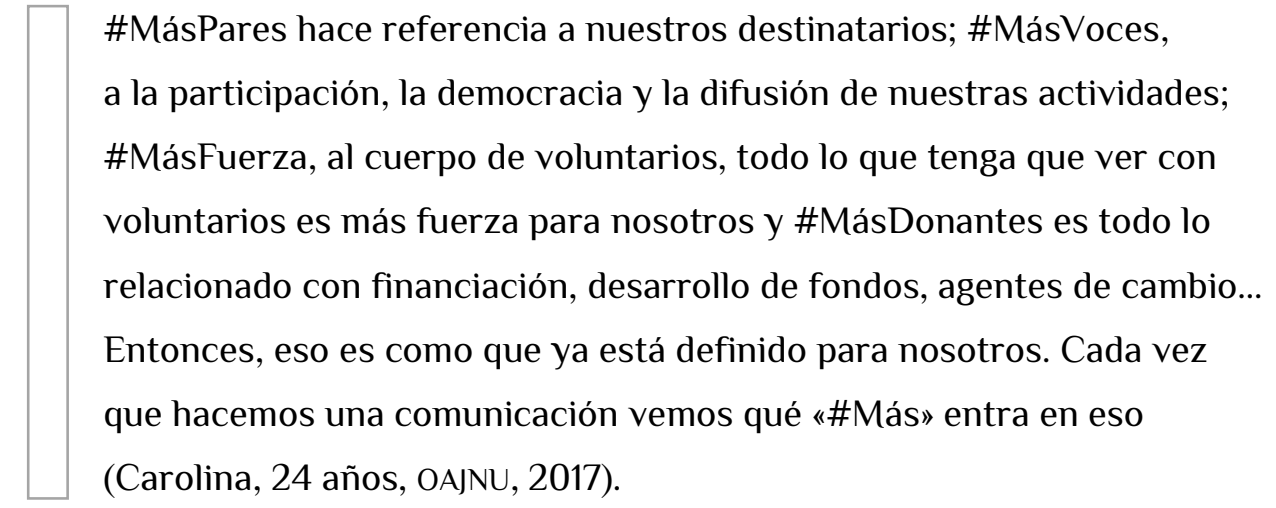

En las interpretaciones de los/as entrevistados/as, Twitter aparece como más dinámico, más instantáneo. Por esta razón, la estrategia de uso es subir fotos e información de lo que ocurre en el momento -en el transcurso de aulas talleres o de reuniones- o replicar información (retuits) que se publica en el perfil nacional de OAJNU Argentina o de otras sedes -la Sede de Salta fue con la que más intercambios tuvieron durante el período de relevamiento- o en las cuentas de los/as propios/as voluntarios/as que comparten las actividades o las expectativas para con la organización; en todos los casos, y siempre que sea posible, incorporando los hashtags dispuestos en el Plan Estratégico 2014.

\section{Lo invisible}

En el caso de Techo, Gabriel (2016) y. luego. Federico (2017) fueron los coordinadores de comunicación de la sede CoCha, acompañados por distintos/as voluntarios/as dentro del área. En el caso de OAJNU, en 2016, la administración de los espacios digitales correspondía a la Secretaría de Promoción, cuya función principal era el manejo de la imagen, la difusión de las actividades y la comunicación interna. A partir de 2017, el área se desdobló y quedó conformada por la Secretaría de Promoción de Derechos, que se encargaba de todo lo interno, 
y por la Secretaría de Comunicación, que tenía a su cargo la comunicación externa, lo que incluía las redes sociales digitales.

De nuestro relevamiento de datos surge que ambas organizaciones otorgan un uso instrumental a los espacios digitales, que ambas poseen manuales de marca definidos y que el grueso de las informaciones que publican en sus páginas oficiales están centralizadas en algunos grupos decisores, en áreas específicas de comunicación o, incluso, como ocurre en Techo, centralizadas en CABA.

De las entrevistas, se desprende que los principales canales para intercambios y diálogos, tanto hacia adentro como hacia afuera de ambas organizaciones, son los correos electrónicos -especialmente Gmail, del que utilizan aplicaciones como Calendar o Drive-, la mensajería privada de las redes sociales, el uso de Whatsapp y, en menor medida, el empleo de Skype y de Hangouts para llamadas; es decir, usos que permanecen invisibles en sus perfiles públicos.

En cuanto a usos, pudo identificarse en la totalidad de las entrevistas el valor superlativo que le otorgan al Drive de Google como herramienta de organización interna, y para el trabajo colaborativo inter-sedes y de gestión. Esto se identificó con mayor énfasis en OAJNU, donde, además de emplear el Drive para poner a disposición de los/as voluntarios/as documentos y materiales útiles, mencionan la costumbre de dejar anotados los puntos más sobresalientes de las reuniones presenciales a modo de recordatorio colectivo. El uso del Drive que realizan en las organizaciones sociales, luego lo trasladan a otros ámbitos cotidianos como el trabajo, el estudio o la ayuda a microemprendimientos familiares.

Los grupos de Whatsapp, en tanto, les permiten organizarse y coordinar cuestiones prácticas en tiempo real: «Por ejemplo, qué colectivo tomamos cuando vamos al barrio; cosas tan simples como esa», ejemplifica Andrea (29 años, Techo). Esta herramienta es nombrada, en algunos casos, como una red social más, que rápidamente se incorporó a las prácticas comunicativas y desplazó a otras.

Al momento de pensar los mensajes que pretenden difundir, los/as entrevistados/as identifican como una característica de los consumos juveniles que «toda la interacción es digital» y que los/as jóvenes realizan varias al mismo tiempo. En palabras de Cristian (28 años, Techo), «los jóvenes no quieren perder el tiempo en una persona, sino tener cincuenta interacciones juntas, lo que les da la opción de filtrar y de ignorarte más fácilmente». Junto con este reconocimiento, aparecen con 
claridad quiénes son los/as usuarios/as de las distintas redes sociales digitales, así como las lógicas de publicación en cada una. Estos registros aparecen, sobre todo, en las apropiaciones de OAJNU, organización que cuenta con distintos perfiles y que, mediante la interacción, va delineando las características del público que lo sigue en cada red. En algunas oportunidades, reciben mensajes de docentes que quieren postular a sus colegios para participar; en otras, son personas que se presentan con «proyectos en mente» y a quienes invitan a sumarse como voluntarios/as, ya que todos los proyectos que se ejecutan deben enmarcarse dentro de la organización.

En este sentido, Facebook, que durante mucho tiempo fue tomado como una red juvenil, ahora es considerada una red más de adultos, habiéndose trasladado el «público joven» a redes como Instagram, Youtube y Snapchat. Las prácticas comunicativas que se vinculan a los/as distintos/as jóvenes también son variadas. Incluso, en las entrevistas, los/as miembros de OAJNU plantean que son una de las pocas organizaciones sociales en la ciudad de Corrientes que piensa tanto el espacio digital, cómo llegar a sus seguidores/as o cómo sumar gente, a pesar de que son prácticas que van aprendiendo «sobre la marcha».

En el caso de Techo también identifican distintos públicos a los que deben dirigirse. Por ejemplo, Rodrigo (31 años, Techo) reconoce un público general para los medios oficiales -como puede ser el grupo de Facebook- y un público integrado por los miembros de la organización y por compañeros/as voluntarios/as de áreas puntuales o de grupos de Whatsapp específicos, para las comunicaciones de tipo interpersonal. Otro público suelen ser los/as vecinos/as, tanto de los barrios donde trabajan como de los que no, lo que les hace plantearse cuestiones vinculadas a la comunicación comunitaria.

\section{Estrategias y recursos más utilizados}

Entre las estrategias identificadas, ambas organizaciones hacen un uso casi diario de sus redes digitales y en sus publicaciones pueden identificarse con claridad los destinatarios de los mensajes. Para ello, recurren a la práctica de etiquetar: a los/as voluntarios/as que estarán o que estuvieron participando de una actividad; a las organizaciones, empresas y fundaciones con las cuales estarán o estuvieron 
trabajando; a los medios de comunicación locales; y a las áreas gubernamentales con las que firman acuerdos, como modo de dejar registro de las actividades. No obstante, el análisis de este recurso dejó en claro que la mayoría de las publicaciones tenían por destinatarios/as a los integrantes del equipo interno; es decir, a los/as propios/as voluntarios/as, ya que las redes sociales les permitían actualizar novedades sobre sus actividades y dar aviso de cambios repentinos en días/horarios de actividades ya pautadas.

A partir de los contenidos publicados en los estados, y de las etiquetas y las fotografías compartidas, se hacen explícitos los actores con los que establecen acuerdos de trabajo, especialmente cuando se desarrollan talleres o charlas informativas. ${ }^{6}$ En ambos casos, a su vez, tienen como práctica habitual reiterar las publicaciones (mismo flyer, mismo texto) días después de del primer posteo. Esto se produce, especialmente, con invitaciones a talleres o a actividades que requieren de inscripción previa, a modo de recordatorio.

Ambas organizaciones cuentan con un área que se encarga de la comunicación y se ocupa de las redes sociales, aunque las estrategias empleadas y los grados de decisión y de compromiso sean distintos. Una diferencia a remarcar se relaciona con los espacios ocupados en Facebook: un grupo, en el caso de Techo, y una fanpage, en el caso de OAJNU.

En Techo la particularidad de contar solo con un grupo en Facebook condiciona la llegada a públicos más amplios. En la práctica, estos grupos funcionan como escenarios de organización / discusión de temáticas puntuales y con personas que forman parte del quehacer de las actividades que el propio espacio aglutina. Desde allí, los/as voluntarios/as producen encuestas, linkean formularios para hacer consultas o para relevar sugerencias (por ejemplo, qué temas debatir en el Encuentro de Voluntarios o qué películas proyectar en el Cine Debate). A su vez, generan «eventos» que sectorizan las conversaciones en torno a actividades puntuales (por ejemplo, «Construcción en Resistencia y Corrientes, mayo 2016, TECHO Co-Cha»).

En Techo, además, una práctica habitual es subir al grupo videos propios con compilados de fotografías sobre momentos específicos (colectas, construcciones, reuniones) o con fragmentos que ofrecen testimonios sobre lo que es ser voluntario/a en la organización o sobre las sensaciones que les quedan luego de 
llevar a cabo actividades masivas (colectas, construcciones), lo que también sirve como puntapié para invitar a nuevos/as voluntarios/as y para dejar asentados los trabajos realizados. El uso del streaming, en tanto, es limitado: solo se emplea dentro de la sede para mostrar resultados de procesos de investigación (como el RAl) o momentos de discusión y debates internos (como los preparativos para la colecta).

Por el contrario, las fanpage, como la que utiliza OAJNU, son espacios de promoción y de difusión de actividades, de ideas o de productos, que abarcan distintos perfiles entre sus públicos. Por lo general, son utilizadas por empresas, por personajes y por organizaciones de diverso tipo.

En la planificación que realizan sobre aquello que publican, aparece un registro de días / horarios, en relación con las estadísticas de picos de conexión por parte de los/as usuarios/as. Ambas organizaciones diagraman y realizan un seguimiento de cuántos usuarios/as siguen cada red y en qué momentos se suman. A partir de estas prácticas, los/as jóvenes resignifican sus usos de las redes sociales como un canal de información y comunicación con otros/as, sobre el cual es válido un aprendizaje que permita saber cómo «explotar» su potencial.

Hay una tabla que dice los horarios en que la gente se conecta más seguido, y yo nunca pensé que en mi vida me iba a preocupar por eso, pero ahora la tengo en cuenta si trato de hacer una publicación y que todo el mundo la vea. También esperamos que todas las publicaciones sigan un hilo conductor, que no sean aisladas; cuidar mucho la cuestión OAJNU. Cuidar el vocabulario, las palabras, los términos. Generar palabras clave que sí o sí tengamos que usar para tal actividad o proyecto. ¿Cómo lo comunico? ¿A través de qué red? Es algo que jamás me iba a poner a pensar (Claudia, 22 años, OAJNU, 2017).

Debatimos mucho sobre las redes sociales y los lineamientos. Tenemos un manual de estilo que es larguísimo, donde se habla desde los colores específicos que tenemos que usar para hacer los flyers hasta qué tenemos que comunicar en cada red social. En Facebook, por ejemplo, no vamos a poner tantas publicaciones y las mismas pueden ser un poco más extensas, más informativas (...), pero también empezamos a debatir que no comunicábamos nada a través de Facebook (Melina, 19 años, OAJNU, 2017). 
A lo largo de la investigación, tanto en el momento de recolección de datos como en la instancia de análisis nos interesó indagar cómo los/as jóvenes perciben la tarea de visibilizar las causas de ambas organizaciones a través de las redes digitales. Al respecto, Cristian (28 años, Techo) menciona que es todo un desafío, porque las redes se volvieron solo una herramienta para invitar a actividades y escasas veces son utilizadas para contar qué están haciendo: «Vos hablás de Techo y no sé, creen que es como que lo hubiera hecho McDonalds ¿entendés?». Ante esto, surgió la necesidad de «mostrar la cara humana de Techo», para enfatizar que es una organización de voluntarios/as que trabajan todas las semanas en los barrios y no reducir todo a cuestiones administrativas. En este sentido, una de las estrategias en las redes implicó contar lo que estaban haciendo, por ejemplo, las bienvenidas y las despedidas a voluntarios/as que asumían roles específicos.

De manera similar, en OAJNU mencionan como una dificultad poder dejar plasmado su trabajo en las redes sociales. «Creo que hacemos política (social), pero cuesta mucho mostrarlo, dejar visible que trabajamos esta problemática y cuesta que los propios voluntarios lo entiendan», señala Melina (19 años, OAJNU).

Sobre las interacciones que logran desde sus redes digitales, Cristian (28 años, Techo) se lamenta: «Vemos resultados pobres, en el sentido de que no está interesando a la gente». Esta es una percepción compartida por otras sedes, así como por integrantes de OAJNU respecto de sus propias redes. Ante esta dificultad, una de las estrategias en Techo fue tomar, a modo de experimentación, una campaña de marketing viral para difundir un Encuentro de Personas (actividad que convoca exclusivamente a voluntarios/as activos/as a compartir todo un fin de semana, a modo de retiro, para poder discutir y proyectar la organización), así como solicitar a fanpages de otras organizaciones y/o actores con los que tienen acuerdos -y que a la vez poseen muchos seguidores- que compartan la información para llegar a un rango más amplio, traccionando contactos y visualizaciones. Esta acción redundó en más inscriptos a la actividad y la publicación fue la que más interacción tuvo durante todo el año en término de comentarios y likes. Frente a esto, Cristian (28 años, Techo) reflexiona: «Ahí me di cuenta que estamos perdidos, y mucho, en esas estrategias de comunicación digital. Necesitamos a alguien pensando en estas cosas». 
Para cada actividad planificada, cuentan con estrategias tanto para instancias en la calle como para las redes digitales. Federico (31 años, Techo) cuenta que en Techo se manejan con listas de verificación. Dentro de esa lista, uno de los puntos a considerar en una construcción masiva es lo que llaman «video de envío». Se trata de un video que cuenta la previa de cada trabajo, para que los/as jóvenes conozcan a los/as vecinos/as con quienes van a construir durante el fin de semana. En los videos que se publican en las redes digitales no se suele mostrar a los/as vecinos/as de los asentamientos para no individualizarlos, aunque sí sus comentarios y sus testimonios.

Por citar un ejemplo, en abril de 2016, luego de la primera construcción masiva del año, publicaron un flyer que tenía por imagen de fondo la fachada de una vivienda de emergencia y, en letras de molde en color blanco, el comentario de una vecina beneficiada: «Estoy muy agradecida por la casa que me hicieron, al igual que todos mis vecinos. Me gusta cómo son como personas, siempre amables y respetuosos. Dalma» Desde la organización, acompañaron el flyer con un texto donde dejaban asentados los logros del fin de semana junto el slogan de la organización «por una sociedad más justa y sin pobreza».

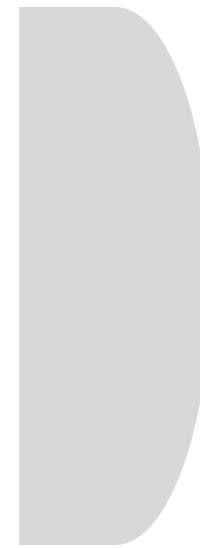

Con tu voluntad y compromiso pudiste transformar la vida de una familia como la de Dalma. Pero no fue la única, sino que son 34 familias que, a partir de este fin de semana, tienen la oportunidad de vivir mejor, de dormir más tranquilos, de no pasar tanto calor ni tanto frío. Después de estos dos días increíbles, 34 familias de Punta Taitalo y de Molina Punta comenzarán a construir un futuro con nuevos proyectos y muchos sueños... $---$

Por involucrarte y participar, por crear y creer en una sociedad más justa y sin pobreza ¡GRACIAS! (Techo, publicación en Facebook, 18/04/2016).

Las formas de comunicarse desde las redes generan distintas percepciones por parte de los/as voluntarios/as de ambas organizaciones. En el caso de Techo, el tono de las publicaciones genera miradas contrapuestas, mientras en algunos casos se las tilda de «serias» -como Federico (31 años, Techo), quien plantea que desde su rol de encargado de comunicación discute mucho estas modalidades e intenta generar un tono más ameno-, en otros, el tono o las modalidades se consideran banales o una forma de hacerse los/as «cancheros/as». 
Al uso de redes lo veo más por el lado de buscar la participación, de convocar gente. Realmente, no me gusta el Instagram de TECHO... Me parece muy desde el amiguismo y de buscar gente por el «canchereo», más que realmente visualizar problemáticas. Facebook no sabría decirte, estoy un poco más colgado. Lo dejé un poco, y si bien entro, no le presto atención (Rodrigo, 31 años, Techo).

Los/as voluntarios/as de ambas organizaciones comparten y replican en sus propios perfiles aquello que se publica en las cuentas oficiales, y también comparten fotografías o escritos propios sobre la organización. Al respecto, mencionan que a veces se yuxtaponen tiempos de mucha actividad en la organización con compromisos externos como tener que estudiar, trabajar o rendir exámenes. "Hay periodos de actividad, de exámenes y demás, en los que no se publica tanto... Por ahí publica el voluntario, pero no la organización» (Carolina, 24 años, OAJNU).

En algunos casos, su presencia en las redes sociales digitales opera como un medio de comunicación externa para informar sobre actividades; en otros, como una herramienta de organización a nivel interno. Esto los/as conduce a tener voluntarios/as encargados de la comunicación y a verse en la obligación de establecer acuerdos sobre cómo mostrarse, qué compartir y cómo difundir. Dicha tarea no se toma a la ligera, sino que los/as compromete a pensar / producir / diseñar textos, imágenes, flyers, fotografías, y a mantener actualizados sus espacios, aunque no necesariamente en tiempo real.

Por ahí, no somos tan organizados en la publicación, nos cuesta mucho eso, porque te lleva mucho tiempo. Las redes sociales son algo de lo que sí o sí tenés que estar pendiente y al momento para hacerlo... Tampoco podemos estar subiendo todos los días un texto, digamos. Es como que cada vez que subimos necesitamos subir algo con contenido, que lleva una preparación; entonces, estar todo el tiempo en el día a día es mucho (Carolina, 24 años, OAJNU).

En este sentido, sus distintos perfiles en redes sociales son complementos de sus actividades presenciales, pero en ellos no queda reflejada la totalidad de las actividades que llevan a cabo ni los territorios que recorren en la ciudad. De hecho, existen dinámicas muy interesantes que se suscitan en sus encuentros cara a cara 
sobre los que muchas veces no quedan registros: debates en los talleres, análisis de las políticas públicas, distribución de roles, juegos, recorridas por la ciudad para realizar los diagnósticos y encuestas, por mencionar algunos ejemplos.

Si bien establecen contactos con medios locales, en ambos casos, una de las principales estrategias consiste en aprovechar al máximo los contactos previos para que actúen como multiplicadores o como viralizadores de las publicaciones y lograr, así, un impacto mayor. ${ }^{7}$

En OAJNU una de las estrategias consiste en hacer visible de manera permanente a aquellas/os voluntarios/as que forman parte de la organización, para destacar sus cualidades personales y desde dónde aportan a la organización. De este modo, ante cambios en la conformación de los equipos o para celebrar los cumpleaños, publican estados y flyers con la foto de los/as voluntarios/as y una breve mención a su trayectoria dentro de la organización, con hashtags vinculados a \#Fuerzajoven y \#CadaVezMás.

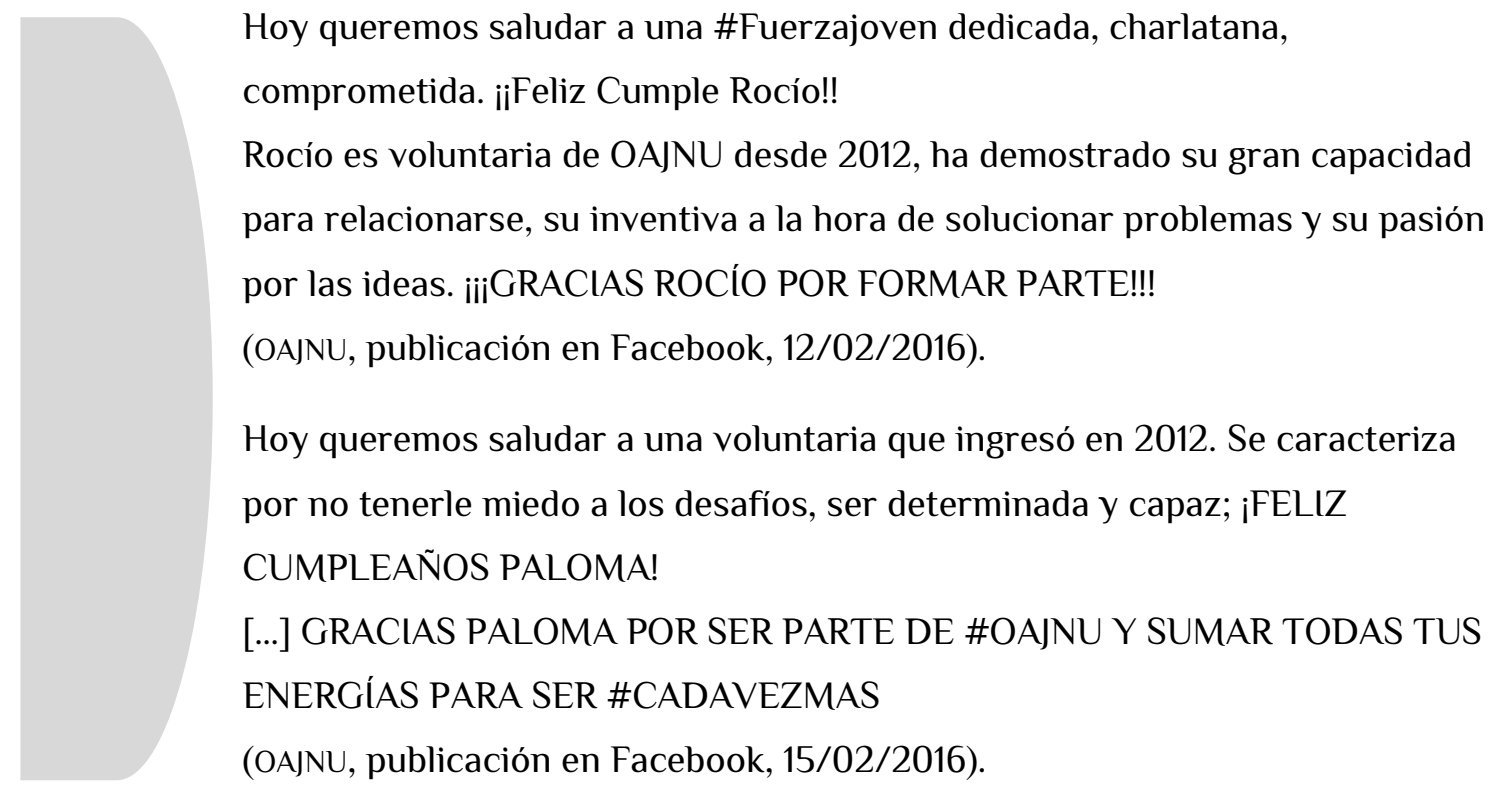

Al momento de codificar el relevamiento, nos llamó la atención que durante los dos años de seguimiento fueron muy escasas las interpelaciones que desde las redes de Techo se realizaron a los «jóvenes» como tales, sino que en primer plano se ponía su calidad de «voluntarios/as». Esto es un punto nodal de diferenciación en el uso de redes que realizan ambas organizaciones, ya que el modo de auto reconocerse en OAJNU es la de ser una organización «de jóvenes, para jóvenes» 
y eso se tradujo en 162 publicaciones que hacían mención explícita a temáticas vinculadas con jóvenes o con juventudes, o mediante las cuales hacían referencia a ser una organización juvenil.

Por el contrario, en el mismo lapso de tiempo, desde Techo solo se hicieron siete menciones explícitas. En dichas publicaciones, convocaron a reunirse con integrantes de juventudes partidarias y expusieron los resultados de dicha reunión; invitaron al taller «Construyendo cambios positivos» organizado por la Cámara Junior Corrientes, y al evento "Queremos ser protagonistas», organizado junto con otras OSC de la ciudad, y convocaron (tres veces, durante agosto de 2017), al Foro de la Juventud, organizado por OAJNU.

\section{Conclusiones}

El trabajo pone de manifiesto que mediante la apropiación de TIC, los/as jóvenes que integran las organizaciones civiles analizadas van incorporando prácticas sociales que les permiten planificar, organizarse, gestionar y difundir, y, a partir de allí, adquirir conocimientos respecto del «saber hacer» comunidad en entornos digitales. Describimos las diferencias existentes en la gestión de la comunicación entre ambas organizaciones: un modelo verticalista (Techo) y otro que, aun con jerarquías, busca fomentar la horizontalidad en el manejo de redes, a la vez que realiza capacitaciones constantes para incorporar el uso de recursos (como los hashtags, en OAJNU) que los/as ayudan a consolidar una presencia en redes en tanto repertorios de la acción conectiva (Reguillo, 2017).

Identificamos que se mueven con soltura en la web social; tienden a generar acciones haciendo uso de las posibilidades de conectividad mediante los hashtags que promueven y solicitando a sus conocidos que los ayuden a «viralizar» aquellos contenidos que publican; tienen noción del valor de la web para vincularse con jóvenes, empresas y organizaciones a nivel global, pero anclados en el territorio, mediante el fomento de usos y de apropiaciones desde un lugar situado social, económica y culturalmente. Las referencias a ser una organización juvenil aparecen explícitamente en OAJNU, constituyéndose, incluso, en su carta de presentación; no sucede lo mismo en Techo, donde el carácter de voluntariado/a es puesto en primer plano. 
Pudimos reconocer los rasgos y las condiciones de apropiación: con entusiasmo y a partir de superar dificultades fueron adquiriendo en la práctica un dominio técnico y cognitivo de las herramientas, las plataformas y las redes sociales con las que llevan adelante las actividades de cada organización (Moreno Gálvez, 2015). Observamos, además, que no solo adoptaron estas herramientas para la organización, sino que las fueron trasladando a otros ámbitos y actividades del día a día como estudiar, trabajar o colaborar con microemprendimientos familiares. En muchas ocasiones, ante la negativa o los recelos a utilizar algunas herramientas (como calendar, Gmail o Whatsapp) fueron apareciendo las voces (y las presiones) de compañeros/as del voluntariado.

Los procesos de apropiación de tecnologías en el marco de las organizaciones los provee de un conocimiento especializado en el uso de redes, vinculado a la organización, la gestión, el reconocimiento de las potencialidades y las limitaciones del espacio digital, la pre-figuración de públicos específicos y de lógicas específicas de publicación en cada una de las redes sociales digitales, entre otros aprendizajes.

\section{Referencias}

Crovi Druetta, D. (2013). Repensar la apropiación desde la cultura digital.

En S. Morales y M. L. Loyola (Comps.), Nuevas perspectivas en los estudios de comunicación. La apropiación tecno-mediática (pp. 11-24). Ciudad Autónoma de Buenos Aires: Imago Mundi.

Feixa, C. y Fernández-Planells, A. (2014). Generación @ versus Generación \#. La juventud en la era hiperdigital. En A. Huertas y M. Figueras Maz (Eds.), Audiencias juveniles y cultura digital (pp. 35-54). Bellaterra, España: Institut de la Comunicació, Universitat Autònoma de Barcelona.

Lago Martínez, S., Méndez, A. y Gendler, M. (2017). Teoría, debates y nuevas perspectivas sobre la apropiación de tecnologías digitales. En R. Cabello y A. López (Eds.), Contribuciones al estudio de procesos de apropiación de tecnologías (pp. 75-86). Rada Tilly, Argentina: Del Gato Gris. 
Lemus, M. (2017). Jóvenes frente al mundo. Las tecnologías digitales como soporte de la vida cotidiana. Revista Latinoamericana de Ciencias Sociales, Niñez y Juventud, 15(1), 161-172. Recuperado de http://revistaumanizales.cinde.org.co/rlcsnj/index.php/RevistaLatinoamericana/article/view/2955

Martín-Barbero, J. (2002). Oficio de cartógrafo. Travesías latinoamericanas de la comunicación en la cultura.

Ciudad de México, México: Fondo de Cultura Económica.

Morales, S. y Loyola, M. I. (2013). La dinámica de la apropiación tecnomediática. En S. Morales y M. I. Loyola (Comps.), Nuevas perspectivas en los estudios de comunicación. La apropiación tecno-mediática (pp. 1-11). Ciudad Autónoma de Buenos Aires, Argentina: Imago Mundi.

Moreno Gálvez, F. J. (2015). Ciudadanía y apropiación tecnológica. Herramientas para el análisis de las prácticas comunicativas de los sujetos en torno a las nuevas tecnologías. Revista Internacional de Pensamiento Político. I Época, (10), 71-83. Recuperado de https://www.upo.es/revistas/index.php/ripp/article/view/3586

Pannunzio, M. F. (2019). Jóvenes, espacios digitales y procesos de socialización política. En T. Artieda, M. J. Simoni y G. Vega (Comps.), Libro de actas de las III Jornadas Intercambio de la Producción Cientifica en Humanidades y Ciencias Sociales (pp. 111-113). Resistencia, Argentina: Facultad de Humanidades, Universidad Nacional del Nordeste. Recuperado de http://hum.unne.edu.ar/publicaciones/invest_posgrado/terceras_jornad as.pdf

Reguillo, R. (2017). Paisajes insurrectos. Jóvenes, redes y revueltas en el otoño civilizatorio. Madrid, España: NED Ediciones.

Wincour, R. (2009). Robinson Crusoe ya tiene celular. Ciudad de México, México: Siglo XXI. 


\section{Fuentes}

OAJNU Facebook (12 de febrero de 2016). Sin título. Recuperado de https://www.facebook.com/OAJNUCtes/photos/a.345927838817932 /952083521535691

OAJNU Facebook (15 de febrero de 2016). Sin título. Recuperado de https://www.facebook.com/OAJNUCtes/photos/a.345927838817932 /952556654821711

Techo Facebook (18 de abril de 2016). Sin título. Recuperado de https://www.facebook.com/groups/voluntarios.corrienteschaco/pos ts/890060954436916

Techo Facebook (31 de octubre de 2016). PRESENTACION RAI. Recuperado de https://www.facebook.com/groups/voluntarios.corrienteschaco /posts/1016144025161941

Notas

1 Durante la investigación, se analizó un total de 1.258 posteos, que se indican de manera desagregada en el «Documento complementario» que acompaña este artículo y en los enlaces allí ofrecidos.

2 La tesis, titulada «óvenes, espacios digitales y procesos de socialización política: los casos de las organizaciones OAJNU Corrientes y Techo Corrientes en el periodo 20162017», fue dirigida por Leonardo Murolo (Universidad Nacional de Quilmes-Universidad Nacional de La Plata), codirigida por María Mercedes Oraisón (Universidad Nacional del Noroeste) y defendida el 11 de marzo de 2021 en el marco del Doctorado en Comunicación de la Facultad de Periodismo y Comunicación Social de la Universidad Nacional de La Plata. Disponible en http://sedici.unlp.edu.ar/handle/10915/117076

3 Al momento de producción del artículo (octubre de 2021), el grupo cuenta con 3.142 miembros. 
4 El Modelo Naciones Unidas Junior se realiza entre establecimientos educativos de la misma ciudad y está destinado a jóvenes de entre 13 y 15 años.

5 Reguillo (2017) caracteriza este recurso a partir de una etnografía realizada en 2011 medio de las protestas del Ocuppy Wall Street, donde manifestantes carentes de micrófonos y de recursos técnicos similares para llevar a cabo las protestas, se organizaron y empezaron a amplificar el discurso de los/las oradores/as a partir de repetir a viva voz aquello que oían. La autora remarca cómo la práctica requiere de la escucha atenta y de un grado de «conexión» con el momento para que el mensaje se amplifique sin distorsiones.

6 Por ejemplo, en Techo, la utilización de aulas de la Facultad de Arquitectura de la Universidad Nacional del Nordeste (UNNE); en OAJNU, el uso de las instalaciones del Club de Regatas Corrientes, el Tenis Club o aulas de la Universidad Católica de Salta (UCASAL).

7 A menudo, los/as voluntarios/as me escribían por privado enviándome mensajes de Whatsapp o via Messenger en Facebook, solicitándome que compartiera en mi muro algunos posteos puntuales, generalmente, propios de campañas de concientización o de actividades que esperaban fueran masivas. Esta dinámica se dio, fundamentalmente, en Techo, donde fui matizando mis vínculos para no perder contacto con informantes clave. 